\title{
Les cours de langues dans le contexte universitaire: quelle place pour la compétence interculturelle à l'ère du numérique?
}

\section{Language courses in the university context: what place for intercultural competence in the digital age?}

\author{
Khalil MoussafiR \\ Université Hassan 1er (Maroc) \\ khalil.moussafir@uhp.ac.ma
}

\begin{abstract}
This article aims to highlight the significant place of interculturality in the language classroom. Indeed, current societies are exposed to profound changes at the individual and collective levels under the effect of digitization. This means that the individual experiences a new socialization, a kind of digital socialization within which the intercultural dimension occupies a central place through linguistic and cultural openness to the other. This situation presents the school with new challenges. Beyond didactic and purely linguistic considerations, language learning takes on its full significance by putting to work the founding concepts of interculturality such as intercultural competence or intercultural communication competence. We will show this from the linguistic programs of international cooperation, in particular those of the partnership between the University Hassan1er in Morocco and the Haute école d'Hénallux in Belgium.
\end{abstract}

Keywords

Intercultural competence, pluralistic approaches, linguistic reinforcement, socio-educational environment.

\section{Resumen}

Este artículo tiene como objetivo aclarar el lugar significativo de la interculturalidad en el aula de idiomas. De hecho, las sociedades actuales están expuestas a cambios profundos a nivel individual y colectivo bajo el efecto de la digitalización. Esto significa que el individuo experimenta una nueva socialización, una especie de socialización digital dentro de la cual la dimensión intercultural ocupa un lugar central a través de la apertura lingüística y cultural al otro. Esta situación presenta a la escuela nuevos desafíos. Más allá de las consideraciones didácticas y puramente lingüísticas, el aprendizaje de idiomas adquiere toda su significación al poner en práctica los conceptos fundacionales de la interculturalidad como la competencia intercultural o la competencia comunicativa intercultural. Lo mostraremos a partir de los programas lingüísticos de cooperación internacional, en particular los de la asociación entre la Universidad Hassan1er en Marruecos y la Haute école d'Hénallux en Bélgica.

\section{Palabras clave}

Competencia intercultural, enfoques pluralistas, refuerzo lingüístico, entorno socioeducativo. 


\section{Introduction}

Dans un monde hyper connecté où la technologie du numérique envahit de manière percutante l'espace public et privé, les barrières géographiques s'amenuisent et le rapprochement des individus des quatre coins du monde s'impose avec tout ce que cela pourrait avoir comme conséquences sur les plans individuel et social. Ces mutations, parfois invisibles à l'œil nu, ont engendré, aujourd'hui, une demande forte sur l'apprentissage des langues étrangères. Ce qui nous met face à la réalité que l'apprentissage des langues se fait également en dehors de l'école et de l'institution pédagogique. Cependant, cet environnement socio-éducatif en perpétuel changement renforce la place de l'école et la met face à un double défi. Accélérer, d'une part, l'intégration du numérique et des technologies d'information et de communication (TIC), comme outils et supports de cours et comme demande sociale urgente. Et d'autre part, assurer la supervision des contenus “numérisés” proposés et le suivi de l'action pédagogique au-delà de l'espace géographique de l'école. Cette nouvelle vocation et ce rôle prégnant attribués à l'institution scolaire et universitaire introduisent des changements d'ordre épistémologique et didactique portant non seulement sur la nature de l'action pédagogique à mettre en œuvre, mais également sur le type de compétences à développer, surtout que les modes d'échanges adoptés vont au-delà des considérations purement linguistiques. "Langue" et "culture" sont "les deux facettes d'une même médaille" (Benveniste, 1976: 263) et l'enseignement d'une langue étrangère ne pourrait en aucun cas se limiter à la dimension linguistique, car 'toute langue véhicule et transmet, par l'arbitraire de son lexique de sa syntaxe, de ses idiomatismes, les schèmes culturels du groupe qui la parle" (Blanchet, 2004: 6).

Notre contribution consiste justement à porter la réflexion sur la compétence interculturelle et ses enjeux didactiques. Nous prenons pour champ de réflexion les cours de langue assurés au sein de l'Université Hassan $1^{\text {er }}$ (UH1), notamment les cours de renforcement en langue française dans le cadre du partenariat international avec la Haute Ecole de Namur-Liège-Luxembourg (Hénallux). Sachant bien qu'au départ les organisateurs ont assigné à cette rencontre des objectifs purement linguistiques, les participants des deux parties, c'est-à-dire les enseignants chercheurs belgo-marocains, les enseignants stagiaires de l'Hénallux et les étudiants marocains de l'UH1, sont unanimes sur les valeurs interculturelles véhiculées dans le cadre de ce programme d'échanges. En effet, c'est l'échange qui est le centre astronomique de la rencontre et il joue un rôle fondamental dans l'intercompréhension et le dialogue des cultures. Cette expérience de terrain permet de vérifier le degré d'efficacité d'un certain nombre d'outils et d'approches didactiques à l'instar des approches plurielles, de la didactique de l'intercompréhension et de l'intégration, de la biographie langagière et de la perspective actionnelle, entre autres, et leur rôle probant 
dans la consolidation de la compétence interculturelle. La conception théorique engagée a mobilisé des domaines plus vastes au-delà de la composante linguistique, pour mieux saisir les contours de la dichotomie langue-culture et sensibiliser les étudiants à l'interculturalité comme vecteur de valeurs de la citoyenneté universelle. Ainsi, notre travail se focalisera, en premier lieu, sur les précisions conceptuelles et terminologiques de l'interculturel, de la compétence interculturelle et les plus-values à tirer de la prise en compte de la compétence à la communication interculturelle. Ensuite, nous proposerons de mettre en perspective l'action interculturelle à partir de pratiques linguistiques en prenant appui sur l'expérience de l'UH1, le dispositif mis en place et la place du numérique. Enfin, nous montrerons que cette ouverture à l'international témoigne de la pluralité et la diversité qui mènent à des concepts novateurs qui auront leurs mots à dire dans l'instauration de la culture de la citoyenneté universelle.

\section{Approche de l'interculturel}

La notion d'interculturel est l'une de ces notions qui exercent une emprise particulière en raison de la diversité de son usage et la portée phosphorescente de sa signification, en ce sens qu'elle continue à séduire les chercheurs et à les stimuler à lui trouver d'autres sens possibles en raison de l'évolution des contacts humains et culturels. En effet, cette notion remonte aux années 1970 et désigne chez les chercheurs américains l'éducation multiculturelle. Pendant ces deux dernières décennies, et surtout depuis le milieu des années 1990, l'accélération de la globalisation et le contexte des firmes et des organisations tournées de plus en plus vers l'international, les interactions interculturelles se multiplient et ne sont plus l'apanage de quelques personnes réfugiées ou poussées à l'immigration. Les contacts avec l'autre (professionnels, fournisseurs, collègues de travail, clients, étudiants...) sont nombreux. Ce qui impose la nécessité de comprendre cet autre de manière scientifique et rationnelle, en dehors de la réductrice vision folklorique, empreinte de clichés et de stéréotypes dominant le contact et les échanges qui en découlent. L'interculturel comme concept porteur de prémisses d'une société internationale en changements et en mutations profonds va marquer des domaines de recherches comme l'anthropologie, les sciences de la communication, l'éduction, la psychologie et les sciences de gestions, etc.

Plusieurs définitions de l'interculturel sont avancées, parmi celles qui ont retenu notre attention, nous citons la définition de Clanet qui met l'accent sur

l'ensemble des processus -psychiques, relationnels, groupaux et institutionnels- générés par les interactions de cultures dans un rapport d'échanges réciproques et dans une perspective de sauvegarde d'une relative identité culturelle des partenaires en relation. (1990: 21) 
Les contacts et/ou les relations entre personnes, groupes, pays ou organisations ne sont pas considérés sous l'angle factuel et sporadique d'analyses généralistes et occasionnelles, mais font l'objet d'études scientifiques régulières, en témoigne l'intérêt croissant à l'égard du concept. Les regards sur l'altérité ont toujours existé, cependant, la prise en compte de ce qui réunit les hommes, et non pas ce qui les sépare, constitue bien l'apanage de l'interculturel, sous l'effet de l'universalisme montant et du relativisme culturel.

Le changement d'angle d'attaque introduit un nouveau mode de relation favorisant l'échange réciproque et l'enrichissement mutuel, au lieu du choc générant conflit et animosité. Le propre de l'action et de l'interaction interculturelles n'est pas de neutraliser les différences culturelles, et encore moins de les superposer ou les annuler, mais d'être capable d'entrevoir comment elles opèrent, se transforment et transforment ceux qui entrent en contact. Il faudrait bien savoir que "[1]'interculturel n'est pas seulement la mise en relation de deux objets, de deux ensembles indépendants et relativement fixes. C'est un phénomène d'interaction où ces objets se constituent tout autant qu'ils communiquent" (Lipiansky, 1992: 192). Culture et communication sont pour ainsi dire les maîtres mots de toute approche de l'interculturel. Celle-ci nous apprend que l'accent doit être mis davantage sur les rapports que le "moi" social ou individuel engage avec autrui plutôt que sur autrui (Abdallah-Pretceille, 1996). Selon cette spécialiste des questions de l'interculturel en pédagogie et éducation, l'innovation majeure que cette notion ramène par rapport à la culture implique trois perspectives à savoir la perspective subjectiviste, interactionniste et situationnelle (Abdallah-Pretceille, 1985).

Pour ce qui est de la première perspective c'est-à-dire subjectiviste, elle est de nature à souligner le caractère unique de l'acquisition de la culture par les individus, chacun selon un parcours qui lui est propre. Cette subjectivité de la culture est souvent intériorisée de façon inconsciente. Ce qui laisse apparaître les divergences lors des contacts entre des individus de cultures différentes. Alors que dans le cadre de la deuxième perspective "interactionniste" l'accent est essentiellement mis sur les interactions des acteurs et en particulier sur le rapport que l'individu entretient avec l'autre que sur l'autre en tant qu'étranger par rapport à nous. Avec tous les clichés et les préjugés socioculturels que cela puisse faire apparaitre. Enfin, la troisième perspective est dite situationnelle car il ne s'agit pas seulement de partir des différences aux niveaux des valeurs et des codes culturels dans l'interaction entre des personnes d'origines culturelles différentes. Lesdites différences relèvent de statuts et d'évolutions historiques, sachant bien que les cultures sont également le fait de l'histoire, avec tout ce que cela présuppose d'événements politique, économique, identitaire et idéologique, etc. Ce qui ne fait que rendre ces interactions complexes. C'est dans cette perspective que Cohen-Emerique tente de redéfinir l'interculturel en tant qu' 
interaction de deux entités qui se donnent mutuellement un sens dans un contexte à définir à chaque fois. C'est un processus ontologique d'attribution de sens et un processus dynamique de confrontation identitaire qui peut malheureusement évoluer vers un affrontement identitaire, une dynamite identitaire. (2000: 172)

Ceci dit, pour faire face aux différents glissements et affrontements non souhaités, les chercheurs, à l'instar des décideurs, inscrivent le processus des interactions interculturelles dans une dynamique positive, que ce soit dans le domaine d'éducation ou dans les autres secteurs d'activités vitaux: dans la coopération et le commerce internationaux et dans les affaires. C'est dans ce cadre que se généralise le concept de "compétence interculturelle".

\subsection{La compétence interculturelle}

\subsubsection{Genèse d'une notion}

De son côté, la notion de "compétence interculturelle" couvre plusieurs domaines d'études qui vont des sciences de communication à l'éducation et de la psychologie en sciences humaines aux sciences de gestion. Pourtant, l'on s'accorde généralement pour reconnaitre l'anthropologue américain Hall, comme le premier à avoir utilisé le concept de "compétence culturelle" en 1959. L'intérêt pour le terme est parti, donc, des Etats-Unis d'Amérique pour inspirer d'autres chercheurs dans le monde et gagner, à l'instar du concept interculturel, d'autres champs d'investigation. Cette diversité d'usage de la notion s'accompagne de diverses acceptions du vocable. Ainsi, nous avons "compétence à la communication interculturelle", “compétence d'intercompréhension", “compétence de médiation interculturelle", etc. selon les domaines d'études.

Le besoin croissant de la compétence interculturelle trouve son justificatif dans l'incapacité des modèles classiques de la communication à assurer l'entente et l'intercompréhension entre deux locuteurs de cultures différentes. En effet, le message produit est interprété par le récepteur selon les codes culturels de ce dernier, et non de ceux de son auteur. Ce qui crée un décalage notoire entre la production du message et sa réception/interprétation et modifie son sens. C'est justement dans ces cas de figures que la compétence interculturelle est requise, à la fois, comme faculté adaptatrice de cadres de références divergents, et comme garante d'interactions réussies. La compétence interculturelle est une soft competence, une compétence différentielle qui n'est pas liée à un métier particulier (Bartel-Radic, 2009), mais elle est sociale ancrée dans un contexte interculturel.

La connaissance d'une langue étrangère est certes une bonne chose, mais connaitre les traits culturels propres à un autre pays, c'est-à-dire propres à une autre culture que la sienne en est certes meilleur. Il ne s'agit pas d'oublier complétement sa culture ou de s'effacer devant la culture étrangère. En effet pour Friedman et Berthoin: "la principale 
compétence interculturelle requise aujourd'hui est la capacité de reconnaître et utiliser les différences culturelles comme une ressource pour apprendre et concevoir une action efficace dans des contextes spécifiques" ((2005: 70); cité dans Bartel-Radic, 2009: 13). C'est tout un travail sur l'empathie qu'il faudrait faire valoir au-delà de toute tendance d'identification, d'adaptabilité ou d'assimilation afin d'aiguiser chez l'individu le sens d'initiative à "sentir très rapidement en quoi son propre comportement peut faire problème, [et l'aider à] s'adapter 'relativement' en tenant compte des codes culturels sans renoncer à sa propre identité" (ibid:: 13).

Mais, suffit-il de connaitre la langue et la culture d'autrui pour s'approprier une compétence interculturelle efficace? Pour plusieurs auteurs, la compétence interculturelle relève de la communication et de la compétence à la communication (Cossette \& Verhas, 1999).

\subsubsection{Compétence en communication interculturelle}

On oublie trop souvent que l'individu est "porteur de culture" (Camilleri, 1989), que les cultures sont véhiculées par des individus et que dans le contexte de la diversité et la multiplicité des cultures, l'être humain est considéré plutôt comme producteur de sa culture et non comme le produit de celle-ci. A partir de ces postulats, les conceptions du rapport à l'autre passent par la remise en question de soi, de sa culture et de sa propre vision du monde pour se rendre à l'évidence que sa culture n'est pas universelle. Pour comprendre l'autre, il est primordial de comprendre que l'on est soi-même étranger par rapport à lui. Et que l'on est face à une personne "porteuse d'une culture" différente.

Par ailleurs, l'expérience des médias a participé de manière incontestable à changer notre perception de l'espace et du temps ainsi que notre appréhension du monde. Dans le domaine d'éducation, cela a influencé profondément les manières d'enseigner et d'apprendre. La confrontation aux langues et cultures étrangères devient quasi quotidienne. Et sous l'effet combiné des moyens d'information et de communication, le jeune d'aujourd'hui, même celui qui se trouve dans les lieux les plus reculés, devient le citoyen de ce que le sociologue américain (McLuhan, 1964) appelait déjà le village planétaire Global Village. Les systèmes éducatifs, notamment au niveau de l'enseignement supérieur sont volens nolens ouverts sur le monde et sur l'autre. Les didacticiens des langues et des cultures sont à cet effet invités à non seulement mettre en place des programmes d'apprentissage des langues, mais à prendre en considération les diversités culturelles afin de faciliter l'intégration des étudiants "étrangers".

On reconnait avec Porcher que la compétence interculturelle n'est: "qu'un aspect d'une compétence culturelle proprement dite" (1995: 61). Sachant bien aussi que la communication interculturelle, comme discipline à part entière, travaille à la fois pour jeter une lumière utile sur les enjeux de contacts et de rencontres entre individus de cultures différentes 
pour les rapprocher par l'établissement de ponts d'intercompréhension et de co-construction $\mathrm{du}$ sens. Ce qui nous encourage à concevoir la compétence à la communication interculturelle en tant qu'habileté à contribuer à l'élaboration collective du sens et à la réussite bilatérale des tâches assignées dans le cadre de ses fonctions.

Il va sans dire qu'avec la compétence en communication interculturelle, nous assistons à un changement de paradigme. L'accent étant mis sur soi dans l'échange et la communication et non pas sur l'autre et sa culture comme objet d'études. Cette compétence consiste en la capacité de s'ajuster et de se réajuster en changeant ses propres critères et ses repères identitaires. Autrement dit, pour pouvoir comprendre la culture d'autrui, il serait prégnant de commencer par la connaissance de sa culture à partir de ses propres capacités d'analyse et de communication dans le cadre de ses pratiques linguistiques. Dans le contexte éducatif, la compétence interculturelle n'est pas assurée par la familiarité avec l'autre culture. Elle est le fait de travail sur les concepts de "la compétence à la communication interculturelle" (Cossette \& Verhas, 1999: 319), de la "compétence d'intercompréhension" ou encore de la "médiation interculturelle" (Geoffroy, 1998).

\section{Pratique linguistique et action interculturelle: place de la culture dans la classe de langue}

Dans cette partie nous nous interrogerons sur la place de la culture dans la classe de langue avec la classique question: peut-on enseigner une langue sans enseigner sa culture? Et quelle place accorde-t-on au culturel et à l'interculturel dans le cadre de l'enseignement numérique des langues étrangères

L'actualisation du concept de l'interculturel dans le discours didactique s'effectue à travers des syntagmes protéiformes de types "didactique de l'interculturel", "management de l'interculturel", "approches de l'interculturel"... Autant d'orientations qui embarrassent les auteurs et concepteurs de programmes et de dispositifs didactiques, quant aux choix d'un enseignement "dépendant" ou "indépendant" de la culture. En effet, l'enjeu 'interculturel' de représentations de l'autre et de sa culture, selon une approche socio-cognitive des manuels de FLE n'obéit pas déjà au principe de l'immanence. Qu'en est-il alors des contenus et programme dédiés à l'enseignement numérique des langues? Nous savons parfaitement bien que les progressions et les ressources pédagogiques en matière de conceptions et d'élaborations n'obéissent pas seulement à des contraintes d'ordre méthodologique. Elles sont le reflet de fluctuations et effervescences survenant sur les plans politique, social et économique. Avec la mise en place de la technologie, l'équation est triptyque et les défis didactiques à relever sont de plus en plus complexe.

A vrai dire, les projets de société et les facteurs liés aux événements historiques pèsent lourds sur les programmes pédagogiques et par là les pratiques de classes. Ils dé- 
terminent les rapports langues/cultures. Aussi, les dispositifs d'enseignement des langues sont tributaires de la problématique de l'identité, ils sont mobilisés, pour ne pas dire manipulés par le politique. Ce qui nous met face au choix de l'enseignement de la langue indépendamment de sa culture. Au Maroc, dans les pays du Maghreb en général, et probablement dans d'autres pays du monde, l'enseignement/apprentissage de la langue étrangère a toujours été fortement inscrit dans une sorte d'"indépendance" linguistique, c'est à dire l'autre versant de l'indépendance politique et économique. Cependant, et en matière de culturalité, le rejet de l'altérité se traduit par le parti pris d'enseigner la langue sans culture. Cette vision réductrice ne favorise pas la dynamique interculturelle. La solution est à chercher dans l'entre-deux. Le rapport à soi passe par la prise en compte du rapport à autrui. Rapport établi, bien entendu, selon l'effort mutuel à déployer pour trouver des points communs en parallèle avec les différences apparentes, car "l'autre est toujours une autre origine du monde et nous sommes à inventer. Et l'être du nous, et l'être même. Au-delà de l'être" (Derrida, 1987: 60).

Dans cet ordre d'idées, l'interculturel paraît comme solution adéquate et réponse aux tenants de l'enseignement de langue sans son ancrage culturel. L'attitude réticente face à la culture étrangère traduit en fait le sentiment d'insécurité linguistique et culturelle. Cependant, les choix didactiques et les orientations épistémologiques en matière de compétence interculturelle ou compétence à la communication interculturelle pourraient-ils se baser sur une approche subjective et intuitive?

\subsection{Choix didactiques et compétence interculturelle}

Sur le plan didactique et méthodologique deux choix se présentent quant à la question d'élaboration de programmes et de conception de manuels ou de ressources pédagogiques. Nous pouvons, dans le cadre du premier choix et selon une approche contextualisée, partir du local pour s'ouvrir sur l'international. De même, il est tout à fait possible, selon les orientations du second choix, de commencer par des supports de dimension internationale pour aboutir, dans une perspective comparative, à la couleur locale. C'est le premier choix qui fait office dans la conception du manuel Cap Université (CU) ${ }^{1}$, support pédagogique adopté au niveau du Maroc dans le cadre de mise en place du dispositif intégré de l'enseignement des langues.

Sur la première de couverture de ce manuel, en plus du titre et d'autres indications (date, du niveau, etc.) figure la mention "module transversal". Cette dernière spécifie la nature même du module et l'inscrit sous le signe de la transversalité et de l'oblique. L'interculturel ici est présent dans ce que laisse entendre la nature du regard réticent, voire

1 Cap Université est un manuel élaboré dans le cadre du Programme d'urgence au Maroc et a été programmé à partir de l'année universitaire 2009/10 pour l'enseignement du français au niveau du cycle de la licence. 
"perçant" sur une langue avec laquelle le jeune marocain entretient un rapport difficile ${ }^{2}$. Cependant, la transversalité mise en avant par la désignation dudit module interroge le type de compétence visé en premier lieu. Autrement dit, une compétence en rapport avec les facultés linguistiques et communicatives de l'étudiant. Rien ne nous empêche d'assigner des objectifs interculturels au cours de langue. De plus, ce qui nous intéresse dans l'étude de la transversalité, c'est surtout le double objectif poursuivi: doter l'étudiant d'outils linguistiques et d'habiletés communicatives nécessaires pour suivre de manière aisée les contenus disciplinaires et le conforter dans son attitude d'autonomie par la nature des activités proposées, surtout celles en lien avec le développement de ses réflexes d'analyse et de son attitude critique.

Dans le manuel CU, chaque dossier se solde par la double-page: "Ici et ailleurs". L'intérêt de ce choix pédagogique ne réside pas seulement dans l'ouverture à l'international, pour enrichir les connaissances de l'étudiant par les informations et les pratiques courantes chez les autres ou dans les autres pays sur les faits et les thèmes étudiés, mais d'inscrire l'action pédagogique dans la pluralité culturelle. Pluralité à considérer comme synonyme d'enrichissement et de valeur ajoutée.

A côté du manuel CU, l'université UH1 a mis en place et en collaboration avec la Haute école d'Hénallux l'outil pédagogique Tremplin pour l'enseignement apprentissage du français langue universitaire au Maroc en 2016. La démarche didactique préconisée dans ce livre est différente de celle mise en œuvre dans le CU, mais elle répond aux mêmes objectifs, c'est-à-dire une remise à niveau linguistique permettant aux étudiants de poursuivre leur cours de spécialité avec aisance. L'intérêt de cette collaboration est double elle constitue: "tout à la fois une opportunité de formation à caractère propédeutique pour les étudiants de l'UH1 et une expérience d'investigation didactique particulièrement riche pour les futurs enseignants de l'Hénallux" (Donckers et al., 2017: 18). De tels propos témoignent d'un travail de terrain de longue haleine par les enseignants chercheurs des deux institutions partenaires (Moussafir, 2017). En effet, l'une des remarques saillantes qui attire, d'ailleurs, l'attention de l'équipe mixte des chercheurs est que:

les enseignants de 1'[UH1], souvent des spécialistes de la littérature ou des lettres françaises, éprouvaient beaucoup de difficultés à utiliser les manuels de la série Cap Université [...] pourtant édités en conformité avec le Cadre Européen Commun de Référence pour les langues (CECR, 2001), et à destination de différentes filières de formation: qui les jugeant ou trop scolaires ou inadaptés pour les grands groupes auxquels ils étaient confrontés ou d'un niveau trop élevé au regard de celui de leurs étudiants, qui regrettant de ne pas y percevoir la méthodologie pour les utiliser efficacement, qui

2 Le rapport à la langue française est qualifié de difficile parce que cette langue rappelle la présence coloniale de la France au Maroc et en même temps, elle assure, aujourd'hui, l'ascension sociale des Marocains étant donné que le français est la langue d'enseignement des filières scientifiques et techniques au niveau du cycle supérieur. En plus, elle domine les secteurs vitaux en économie, en échanges commerciaux, finances, diplomatie, affaires ... 
estimant encore que les lacunes à combler prioritairement sont d'ordre linguistique, grammatical ou lexical [...] (Donckers et al., op. cit.: 17)

Ainsi, des cours de français ont été programmés les matinées avec des groupes de 25 à 30 étudiants, alors que les après-midis sont réservées à des ateliers thématiques portant sur les pratiques de l'oral, la citoyenneté, la chanson francophone, les films, les jeux oraux $^{3} \ldots$

Le propos ici n'est pas de présenter laborieusement cet outil, ni de nous étaler sur cette expérience unique regroupant deux établissements universitaires: l'un du nord et l'autre du sud, reliés par les mêmes soucis et ambitions scientifiques et académiques. D'ailleurs, Tremplin n'est qu'un élément parmi d'autres dans le cadre d'un dispositif multidimensionnel.

\subsection{Un dispositif multidimensionnel au service de la langue française}

Le projet de collaboration entre l'UH1 et l'Hénallux comporte plusieurs volets à savoir l'organisation de cours de renforcement en français, l'élaboration de ressources pédagogiques, la formation des enseignants de langue à la didactique, l'exploration du numérique dans la classe de langues, l'encouragement de la mobilité des étudiants et du personnel pédagogique et administratif, etc. Nous joignons en annexe (Annexe 1) une fiche technique du déroulement de ce programme d'échanges avec des précisions sur les objectifs assignés au projet (notamment la mise en application du Consortium, le contexte, l'équipe d'accompagnement ...).

Pour ce qui est du volet numérique, les deux institutions ont réussi dans le cadre du programme Erasmus international à élaborer des parcours d'apprentissage. Plusieurs options ont été essayées en fonction des plates-formes d'apprentissage disponibles et opérationnelles au niveau des deux institutions partenaires Moodle, Claroline... Finalement, le choix a porté sur l'exploitation des murs de collaborations multimédias et sur Padlet plus précisément. La philosophie de ces murs virtuels est de "faire tomber les murs de la classe". Ce sont des outils d'apprentissage qui offrent de nouvelles possibilités en termes de généralisation de l'accessibilité aux ressources pédagogiques. Ils renforcent l'autonomie des étudiants par le biais de pratiques pédagogiques inclusives et facilitent l'échange, le classement, la lecture et le traitement des documents numériques à des fins d'enseignement et d'apprentissage. De plus, cet outil numérique permet de regrouper des images, des textes, des sons, des liens Internet et des vidéos sous la forme de vignettes appelées posts. Nous tenons à souligner le rôle prégnant qu'introduit cet outil dans la classe et toute la dynamique des échanges entre

3 Pour des informations plus détaillées sur le projet de partenariat entre l'UH1 et l'Hénallux, se reporter à l'article de Jean Donckers et al. intitulé "État des lieux d'une recherche-action: enseigner le Français Langue Universitaire dans une perspective actionnelle au Maroc", publié dans la revue le Langage et l'Homme aux éditions l'Harmattan. 
les étudiants qui s'impliquent plus dans la co-construction du "mur". Toutefois, l'enseignant veille au bon déroulement des travaux par la maitrise de la règle du jeu en expliquant les modalités du déroulement des activités, étape par étape.

\subsubsection{Le mur collaboratif: mutualisation et organisation}

Dans la phase d'élaboration de l'outil Padlet, il très important d'insister sur les deux principes de base de son fonctionnement optimal, à savoir la mutualisation et l'organisation. En effet, les murs collaboratifs permettent aux étudiants, de façon générale, le partage et l'échange des résultats de leurs recherches sous forme de notes ou de remarques ou même de productions de documents (comptes-rendus, rapports, audios, vidéos...). Ici la mutualisation prend le sens restreint d'échanges centralisés entre un groupe limité, dans lequel tout le monde connait tout le monde, et où la quantité d'informations partagées dépend de l'effort collectif. Chacun peut ramener de nouvelles ressources et accéder à celles des autres.

Pour ce qui est de l'organisation, elle est du ressort de l'enseignant. En effet, ce dernier doit formuler des consignes claires sur les tâches à accomplir par les étudiants afin que le travail se déroule de manière satisfaisante. Les délais de dépôts de ressources ou de remise des travaux sont à délimiter pour qu'il n'y ait pas de relâchement ou du désintérêt. La contribution de chacun des intervenants est à définir dès le départ, car dans le travail de groupes, il y a toujours ceux qui ont tendances à "se cacher derrière les autres". De plus, le travail de l'organisation fait appel à la didactique en ce sens que l'enseignant fournis tous les documents nécessaires à la réalisation des tâches (grilles, formulaires, supports, références bibliographiques ...). Ce qui participe à la création de la dynamique, l'interactivité et la motivation nécessaires à l'efficacité des tâches engagées. Le défi majeur dans l'enseignement numérique en classe de langue est celui de maintenir son public intéressé et emporter son adhésion. Sous Covid-19, et suite à la suspension des cours en présentiel, le passage brusque à l'enseignement distanciel a donné du fil à retordre aux enseignants, surtout pour ceux qui ne tablaient pas sur cette forme d'enseignement.

\subsubsection{Contributions des enseignants et didactique de la "digitalisation"}

A parler du travail des enseignants et surtout de leurs contributions dans le cadre du programme de "digitalisation des parcours" susmentionnés, nous reproduisons ici la fiche descriptive du parcours intitulé "Elaborer un écrit de type universitaire: le compte rendu", alors que le "canevas type" adopté par les enseignants chercheurs dans la mise en œuvre de leurs parcours, nous le proposons en annexe (Annexe 2). Cette fiche donne les informations précises sur la tâche à réaliser et sur le contexte pédagogique, avec des précisions sur le public cible, le scénario actionnel, c'est-à-dire le type d'activités engagées ainsi que les documents et les ressources mobilisés. Il est très important d'indiquer même de manière 
approximative, le temps qui sera alloué par les étudiants hors classe à l'ensemble des travaux effectués pour la réalisation de la tâche finale. Enfin, cette fiche se solde par une activité réservée à l'évaluation.

\begin{tabular}{ll}
\hline Tâche à réaliser & Elaborer un écrit de type universitaire: le compte-rendu \\
\hline Contexte pédagogique & \\
\hline Public ciblé & Etudiants de 1ère année de licence \\
\hline scénario actionnel & Formation de 4 ateliers: \\
& - Technique de lecture efficace \\
& - Niveaux de lecture \\
& Recherche documentaire et visite de la bibliothèque \\
& - Terminologie \\
\hline Durée en présentiel & $8 \mathrm{H}$ \\
\hline $\begin{array}{l}\text { Durée estimée des travaux effec- } \\
\text { tués par l'étudiant hors classe }\end{array}$ & $2 \mathrm{H}$ \\
Évaluation: &
\end{tabular}

Figure 1: exemple de fiche descriptive des cours.

\section{L'ouverture à l'international comme gage de pluralité}

La conception unidimensionnelle du culturel, qui sous-tend les pratiques didactiques, repose essentiellement sur l'acception du terme culture, comme relevant d'une identité vécue et partagée comme collectivement "homogénéisante". Ce qui est loin de la réalité des sociétés actuelles marquées par la pluralité linguistique et la diversité culturelle. A ce propos Martine Abdallah-Pretceille ne manque pas de souligner qu'

[au] sein de chaque groupe voire au sein de chaque individu, on constate une pluralisation de plus en plus forte. L'hétérogénéité est devenue le dénominateur commun de tous les groupes, que ceux-ci soient nationaux, sociaux, religieux ou ethniques. (2010: 10)

L'individu n'est pas le produit de sa culture. Il participe lui-même à sa construction au sein même de la diversité. Et comme en théories de communication, le processus culturel/ interculturel est interactif et participatif. La participation est une forme de communication. Cette dernière est un processus basé sur la négociation et le partage, qui vise le consensus. Les modèles économiques actuels convergent vers un modèle plus ouvert et équitable, et donc, pluriel de l'homo communican, c'est-à-dire l'homme du troisième millénaire, capable de communiquer dans plusieurs langues. 
Cette ouverture sur l'autre est une fenêtre sur la mondialisation. La technologie numérique, comme "nouvelle fenêtre" sur le monde, est en passe de révolutionner les rapports sociaux à l'instar du "petit écran" durant les années 1960 du siècle passé. Contrairement à une idée reçue, la levée des barrières douanières et l'ouverture des frontières ne participent pas à la globalisation du modèle unique, elle:

[...] favorise et multiplie les contacts, les lectures, les rencontres et entraîne une ouverture des identités. Chaque individu, même le plus casanier, est, par ses lectures, par la télévision, par internet, etc. en contact avec le monde entier. L'étrangéité est devenue quotidienne et proche. Chaque individu construit son identité selon des modalités de plus en plus différenciées en s'appuyant sur des exemples extérieurs à son groupe de naissance. (Abdallah-Pretceille, op. cit.: 10)

\subsection{Transaction numérique et vie sociale "virtuelle"}

L'usage intensif de la technologie informatique et du numérique est de nature à générer des comportements convergents au sein de groupes sociaux divergents. A l'isolement physique et réel et à l'individualité montante dans nos sociétés, correspond la vie "sociale" virtuelle de chaque individu au sein de la même famille. On dirait que chacun cherche son nouveau groupe d'appartenance. C'est à ce niveau que l'école peut jouer son plein rôle en éveillant le sentiment de citoyenneté universelle et du vivre ensemble.

Rappelons que l'individu ne possède pas une, mais plusieurs identités. Cependant, dans la conception réductrice de l'identité, souvent confondue avec appartenance, l'approche didactique basée sur le socioculturel focalise sur le singulier, le même et ignore les dimensions liées à l'universalité, à l'altérité et à l'interculturalité à faire valoir. Les représentations des langues sont liées aux réponses qu'on est censé donner aux questions: quels rapports y a-t-il entre l'ici et l'ailleurs? Comment définir les relations entre le soi et l'autre? Comment résoudre les écarts entre les attentes et la réalité?

En fait, il n'y a de réalité sociale qu'à travers les représentations que l'on se fait de cette réalité, et que celle-ci nous induit à élaborer une construction "interculturelle" du monde. Elle ne peut être basée que sur les réalités du multilinguisme et du multiculturalisme. Construction qui, rappelons-le, devrait être conçue dans la vision de J.J. Rousseau par le choix de supports d'illustrations qui ne se prêtent ni la confusion ni la contradiction, car “Quand vous leur donnez [c'est-à-dire les apprenants] des préceptes qui se contredisent, quel fruit espérez-vous de vos soins?” (L’Émile, livre second: 80). 


\subsection{Environnement numérique et nouvelles conceptions de l'apprentissage}

Le fait que nous soyons façonnés par nos outils peut sembler, à première vue, un étrange principe de conception et d'apprentissage. Pourtant, cela a l'avantage de nous rappeler un principe classique que l'apprentissage ne se produit que dans un contexte et qu'il est influencé par l'environnement. En termes simples, les outils d'apprentissage font la différence.

Cet aspect contextuel de l'apprentissage -se produisant uniquement à travers un processus d'interaction d'une personne avec l'environnement- est un élément conceptuel clé des théories d'apprentissage. Les outils numériques d'apprentissage font de plus en plus partie de notre environnement. Un environnement d'apprentissage dans lequel tous les étudiants et tous les professeurs sont équipés de moyens technologiques efficaces et performants. Ces outils créent un contexte qui est transformé et imprégné de puissants outils d'apprentissage psychologique. Ils ont radicalement changé les modèles de communication traditionnels et les relations entre les apprenants et les professeurs.

Au départ, les professeurs n'étaient pas préparés au changement et ont été réticents face à cette dynamique d'apprentissage et de relations créées par ces outils, et bon nombre d'entre eux ont encore du mal à s'adapter. La raison expliquant cette réticence, pour ne pas dire rejet, est à chercher dans cet environnement imprégné de ces outils, et où le membre du corps professoral passe du centre de classe dans les modèles classiques de communication, à la périphérie.

Un deuxième impact considérable de ces outils est la facilité avec laquelle les étudiants peuvent personnaliser leurs propres expériences d'apprentissage, car les cours n'ont plus de limites de contenu surtout avec l'arrivée sur scènes des MOOC, de YouTube et autres formes de cours. Les étudiants vivent et se déplacent désormais au sein de la communauté avec la puissance de création des réseaux et de plates formes comme Twitter, Facebook et la messagerie instantanée... L'accès aux outils de communication, n'importe où et à n'importe quel moment, permet aux étudiants de sortir facilement de la structure de cours organisés.

De plus, les outils mobiles et la technologie nomade facilement disponibles prennent désormais en charge l'accès et la circulation des informations en temps réel, ce qui permet aux événements actuels, aux perspectives mondiales et aux ressources lointaines d'être mises en relief immédiatement. Chaque intervention d'un membre du corps enseignant est sujette à une contestation ou à une confirmation de la part d'une personne qui apporte des idées et du contenu qui pourraient lui être assez inconnus et qui défend et soutient ses propres modèles mentaux. L'apprentissage tout au long de la vie prend certainement un nouveau sens maintenant.

Il convient également de noter que les processus de recrutement et de promotion des 
enseignants chercheurs s'effectuent généralement sur la base de la recherche et de la maitrise des contenus, indépendamment du fait pratique que la jeune génération de professeurs consacre aujourd'hui autant de temps à l'usage et à l'appropriation de la technologie qu'à la recherche. Ce qui se répercute nécessairement sur leur enseignement et modes de travail.

Le résultat est que l'on enseigne généralement comme on l'avait appris. Cela n'a pas été trop problématique dans le passé, mais la prolifération de nouvelles technologies et de nouveaux environnements tels que l'apprentissage en ligne ou hybride et la montée en puissance de la nouvelle génération d'étudiants qu'on pourrait désigner comme les "natifs" du numériques, nous mettent face à de nouveaux défis pédagogiques. Les étudiants sont, en fait, à l'aise avec les communications numériques et digitales. Plutôt que de vouloir écouter et suivre des conférences, ils veulent faire et créer des contenus et des ressources.

En définitive, les réflexions sur les méthodes d'apprentissage nous éclairent de manière significative sur les processus impliqués dans l'enseignement et l'apprentissage. La connaissance des modes et de principes d'apprentissage peut aider à guider notre conception afin d'assurer un fonctionnement efficient. Chaque étudiant est à la fois un cerveau et un caractère uniques dans sa structure et ses expériences cumulées. Nous vivons et nous nous rappelons tous des événements un peu différemment des autres. Cette richesse de perspective est à la fois un défi et une puissante force créatrice. La combinaison du caractère unique de chacun parmi nous et de la richesse de la perspective plaide de manière convaincante pour un enseignement-apprentissage pluriel et diversifié en combinant connaissances, contenus et compétences et en plaçant la communauté au sein du processus interculturel.

Enfin, nos environnements d'apprentissage, que ce soit en présentiel dans le campus ou en ligne, ou encore mixte et hybride, sont les endroits où l'enseignement et l'apprentissage structurés ont lieu. Tout comme nous évaluons et repensons les processus d'enseignement et d'apprentissage entre le corps enseignant et les étudiants, nous sommes également invités à prospecter et superviser les environnements dans lesquels l'enseignement et l'apprentissage se produisent, surtout que ces environnements sont de plus en plus ouverts à la technologie et au numérique. A préciser que les conceptions retenues et les outils sélectionnés pour soutenir les processus d'apprentissage et l'action pédagogique sont déterminés en termes de pluralité et de diversité afin que les cerveaux uniques que nous sommes chargés de nourrir se développent en symbiose et en cohérence avec cette pluralité et acceptent l'interculturalité comme partie prenante du processus d'apprentissage et comme règle du jeu. 


\section{Conclusion}

En guise de conclusion, nous avons montré que les mutations profondes et les changements structurelles des sociétés actuelles sont porteurs de prémisses d'une société internationale dans laquelle l'interculturel occupe une place centrale, selon une double dimension. Il est à la fois une faculté adaptatrice de cadres de références divergents, et une clé garante d'interactions réussies. Nous avons vu également qu'avec la compétence à la communication interculturelle, nous assistons à un changement de paradigme. L'accent n'étant plus mis sur l'autre et sa culture comme objets d'études, mais sur soi dans l'échange et la communication. L'individu n'est pas considéré comme le produit de sa culture, mais comme producteur de celle-ci. C'est pourquoi dans les contextes éducatifs, il faudrait sortir, dans le cadre de l'approche interculturelle, de la vision que la compétence interculturelle est assurée par la familiarité avec la culture de l'autre. Celle-ci se développe par le biais du travail sur les concepts de la compétence à la communication interculturelle, de la compétence d'intercompréhension ou encore de la médiation interculturelle. L'efficacité du travail de l'interculturel s'avère, surtout dans les programmes d'échanges internationaux, à l'instar du partenariat entre l'Université Hassan 1er du Maroc et la Haute école d'Hénallux de Belgique, une nécessité de premier ordre, même si les objectifs assignés à ces programmes pourraient être, au départ, purement linguistiques. En un mot, la classe de langue, surtout avec la montée du digital et l'ouverture à l'international, a tout à gagner en faisant de l'interculturel un enjeu majeur du développement individuel et social. Nous avons montré également, même de manière synthétique et schématique, que les programmes internationaux de mobilité estudiantine, d'échanges pédagogiques et de renforcement linguistique sont les lieux privilégiés de travail et de développement de l'interculturel comme enjeu majeur et vocation universelle de l'enrichissement mutuel. Au-delà de la vision superficielle et sporadique, l'interculturel est à concevoir comme un élément fondamental, une partie prenante, dans les curricula, notamment en enseignement supérieur. À cet effet, ne serait-il pas judicieux de prévoir, à titre de proposition, une entrée strictement réservée à l'interculturel comme descripteur dans les modifications introduites dans le Volume complémentaire du Cadre européen commun de références pour les langues de 2018? ou du moins comme élément du descripteur dans les modifications réservées au pluriculturel dans ledit document? 
Anales de Filología Francesa, n. ${ }^{\circ}$ 29, 2021

KHALIL MOUSSAFIR

\section{Références bibliographiques}

Abdallah-Pretceille, Martine 1996. Vers une pédagogie interculturelle. Paris: Anthropo.

Abdallah-Pretceille, Martine. 2010. "La pédagogie interculturelle: entre multi-culturalisme et universalisme" in Recherches en Education, n 9, novembre.

BARTEL-RADIC, Anne. 2009. "La compétence interculturelle: état de l'art et perspectives" in Management international/Gestin Internacional / International Management, $\mathrm{n}^{\circ} 13(4), 11-$ 26: $<$ https://doi.org/10.7202/038582ar $>$ [21/05/2021].

Blanchet, Philippe. 2004. "L'approche interculturelle en didactique du FLE" in Cours d'UED de Didactique du Français Langue Étrangère de 3e année de Licences Service Universitaire d'Enseignement à Distance Université Rennes 2 Haute Bretagne: <https://didac-ressources.eu/wp-content/uploads/2017/09/pdf_Blanchet_inter.pdf $>$ [30/05/2021] .

CAmilleri, Carmel \& Cohen-Emerique, Margalit (dir.). 2002. Chocs de cultures: concepts et enjeux pratiques de l'interculturel. Paris, L'Harmattan.

Clanet, Claude. 1990. L'interculturel. Introduction aux approches interculturelles en Éducation et en Sciences Humaines. Toulouse, Presses universitaires du Mirail.

Cosette, Marie-Nicole \& Verhas, Michel. 2007. "Formation à l'interculturalité en contexte de coopération internationale: une perspective communicationnelle" in Revue des sciences de l'éducation, volume 25, $\mathrm{n}^{\circ}$ 2, 319-338: <https://www.erudit.org/fr/revues/rse/1999-v25n2-rse1836/032003ar/> [11/05/2021].

Derrida, Jacques. 1987. Psyché. Inventions de l'autre. Paris, Galilée (coll. La philosophie en effet), 11-61.

DonCKers, Jean. et al. 2017. "État des lieux d'une recherche-action: enseigner le Français Langue Universitaire dans une perspective actionnelle au Maroc" in Le Langage et l'Homme, 2017-2. Paris, L'Harmattan, 13-26.

Friedman Victor \& Ariane BERTHOIN-ANTAL. 2005. "La réalité: une approche de la théorie de l'action à la compétence interculturelle": <https://doi.org/10.1177/1350507605049904>

Geoffroy, Christine. 2001. La Mésentente Cordiale. Paris, Grasset/Le Monde.

LIPIANSKI, Edmond-Marc. 1992. "La communication interculturelle" in Benoît, Denis (dir.). Introduction aux sciences de l'information et de la communication. Paris, Editions d'Organisation, 187-216.

Marvellaki, Afroditi; Moussafir, Khalil; Donkers, Jean; Azmi, Kamal et Nadia LaRHRISSI (dir.). 2016. Tremplin pour l'enseignement/apprentissage du Français Langue Universitaire au Maroc. UH1er et HENALLUX.

MousSAFIR, Khalil. 2017. "La perspective actionnelle pour contourner les écueils liés à l'hétérogénéité des compétences universitaires, réflexions sur le projet pilote de l'université Hassan 1er et la Haute école Hénallux" in Le Langage et l'Homme, 2017-2. Paris, L'Harmattan, Paris, 27-40. 
Anales de Filología Francesa, n. ${ }^{\circ}$ 29, 2021

LES COURS DE LANGUES DANS LE CONTEXTE UNIVERSITAIRE: QUELLE PLACE POUR LA COMPÉTENCE...

Porcher, Louis. 1995. Le français langue étrangère: émergence et enseignement d'une discipline. Paris, CNDP Hachette-Education.

Rousseau, Jean-Jacques. 1762. Émile ou de l'Education. Livres I, II et III, édition électronique réalisée par Jean-Marie Tremblay: $<$ https://ddd.uab.cat/pub/1611/1611_a2015n9/1611_ a2015n9a7/emile_de_education_1_3.pdf $>[11 / 05 / 2021]$. 


\section{Annexe 1}

Contrat Programme Lié à la

“3e édition de la QUINZAINE DU FRANÇAIS" désignée par "Université d'Automne"

Organisée du 05 au 18 octobre 2015 à l’Université Hassan 1er de Settat

Entre

L'Université HASSAN 1er et les HAUTES ECOLES représentées par:

Catégorie pédagogique - Départements de Bastogne et de Malonne - HENALLUX

Catégorie pédagogique de Virton - HAUTE ECOLE ROBERT SCHUMAN

\section{Mise en application du Consortium concernant le Français Langue Etrangère Se- conde}

Le présent contrat programme est articulé sur le Consortium concernant le Français langue Etrangère Seconde, ce dernier avenant à chaque convention cadre établie entre l'Université Hassan $1^{\text {er }}$ (UH1) et chaque institution partenaire.

Il explicite le cadre général du déroulement de la $3^{\mathrm{e}}$ édition de la Quinzaine du français ainsi que les modalités d'application de la prise en charge et de l'intervention des professeurs stagiaires et des enseignants des deux Hautes Ecoles partenaires dans le présent projet mis sur pied en collaboration avec l'Université Hassan 1er à Settat (Maroc).

\section{Contexte}

Suite à des rencontres entre les responsables des deux institutions, il est apparu, entre autres, qu'une collaboration entre les deux institutions était non seulement possible, mais souhaitable. Les activités d'enseignement des étudiants de l'Université HASSAN $1^{\text {er }}$ sont dispensées en français, quand les étudiants ne maitrisent pas tous, tout à fait la langue française. Et même si les programmes prévoient quatre modules ( $4 \mathrm{x} 48 \mathrm{~h}$ ) de langue et de communication durant les deux premières années, la difficulté de maitrise du français reste préoccupante, surtout que les étudiants sont affectés dans des groupes de 200 à 300 étudiants. C'est dans ce contexte que s'inscrit ce projet de stage des étudiants de l'HENALLUX auprès des étudiants de la $1^{\text {re }}$ année de la faculté de Sciences économiques et juridiques de 1'Université Hassan $1^{\text {er }}$ à Settat (+ de 1400 étudiants et dont le niveau se situe majoritairement entre A2 et B1). Ce stage s'inscrit dans la "Quinzaine de la langue française". 


\section{Equipe d'accompagnement du projet}

\begin{tabular}{lll}
\hline $\begin{array}{l}\text { Organisation } \\
\text { générale }\end{array}$ & $\begin{array}{l}\text { El Mostapha Oualim (Hassan 1 } \\
\text { oualim.elmostafa@gmail.com }\end{array}$ & $\begin{array}{l}\text { Jean Donckers (Henallux) } \\
\text { jean.donckers@skynet.be }\end{array}$ \\
\hline $\begin{array}{l}\text { Responsables } \\
\text { pédagogique } \\
\text { pour le FLE }\end{array}$ & $\begin{array}{l}\text { Khalil Moussafir moussafir07@ } @ \text { Aphrodite Maravelaki } \\
\text { aphrodite.maravelaki@henallux.be }\end{array}$ \\
\hline $\begin{array}{l}\text { Responsables } \\
\text { pédagogiques }\end{array}$ & $\begin{array}{l}\text { Azmi kamal kamaldroits@gmail. Béatrice Colignon } \\
\text { beatrice.colignon@henallux.be }\end{array}$ \\
$\begin{array}{l}\text { pour les } \\
\text { ateliers de }\end{array}$ & $\begin{array}{l}\text { Mustapha Meski } \\
\text { phameski785@yahoo.fr }\end{array}$ & $\begin{array}{l}\text { musta- Vincent Noël vincent.noel@henallux.be } \\
\text { Philippe Crucifix philippe.crucifix@he- } \\
\text { nallux.be }\end{array}$ \\
\hline
\end{tabular}

\section{Dates}

Le séjour de la quinzaine se déroule du dimanche 4 octobre au dimanche 18 octobre 2015. Le stage en lui-même se déroule du lundi 5 octobre au vendredi 16 octobre 2015.

\section{Calendrier général}

\begin{tabular}{|c|c|}
\hline dimanche 4 & $\begin{array}{l}\text { Arrivée en fin de journée à Casablanca } \\
\text { Transfert en autocar vers la Résidence de l'Education nationale, à } \\
\text { SETTAT }\end{array}$ \\
\hline lundi 5 & $\begin{array}{l}\text { Accueil à l'université, séance académique et colloque d'études à } \\
\text { destination des professeurs stagiaires, des enseignants de l'Univer- } \\
\text { sité et autres. }\end{array}$ \\
\hline $\begin{array}{l}\text { du mardi } 6 \text { au ven- } \\
\text { dredi } 9\end{array}$ & $\begin{array}{l}\text { Cours de FLE et ateliers de citoyenneté à destination des groupes } \\
\text { d'étudiants de } 1^{\text {re }} \text { année inscrits à la quinzaine de la langue française. }\end{array}$ \\
\hline weekend du 10 et 11 & Visites culturelles organisées par l’Université Hassan $1^{\text {er }}$ \\
\hline $\begin{array}{l}\text { du lundi } 12 \text { au jeu- } \\
\text { di } 15\end{array}$ & $\begin{array}{l}\text { Cours de FLE aux groupes des étudiants de } 1^{\text {re }} \text { année de l'Université } \\
\text { Hassan } 1^{\text {er }}\end{array}$ \\
\hline vendredi 16 & $\begin{array}{l}\text { Matin: Evaluation des étudiants de la quinzaine par les professeurs } \\
\text { stagiaires de FLE (par groupe classe) } \\
\text { Après-midi: exposition des travaux réalisés dans les ateliers de Ci- } \\
\text { toyenneté }\end{array}$ \\
\hline samedi 17 & $\begin{array}{l}\text { Visite culturelle organisée par l'Université Hassan 1 }{ }^{\text {er }} \text { (Casablanca: } \\
\text { grande mosquée, vieux quartiers...) }\end{array}$ \\
\hline dimanche 18 & $\begin{array}{l}\text { Transfert en autocar vers l'aéroport de Casablanca et retour en Bel- } \\
\text { gique en fin de journée }\end{array}$ \\
\hline
\end{tabular}




\section{Annexe 2}

“Canevas type" adopté pour la mise en ouvre de parcours par les enseignants

\section{Séquence 1}

Leçon 1: Traiter l'information (lire globalement un document et sélectionner les informations)

\begin{tabular}{|c|c|c|c|}
\hline & Justification pédagogique & Déroulement & Ressources \\
\hline 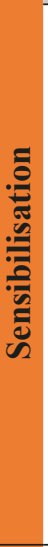 & $\begin{array}{l}\text { Sensibiliser les étudiants } \\
\text { au fait que la réalisation } \\
\text { d'un bon compte-rendu } \\
\text { suppose avoir bien lu ou } \\
\text { entendu les 'textes' de } \\
\text { départ et être capable } \\
\text { d'écrire ou d'en dire l'es- } \\
\text { sentiel dans le genre de } \\
\text { production adéquat à la } \\
\text { situation: réunion- } \rightarrow \text { PV } \\
\text { de réunion; stage- } \rightarrow \text { rap- } \\
\text { port; ... }\end{array}$ & $\begin{array}{l}\text { - Visionner les images projetées } \\
\text { avec la consigne: de quel genre } \\
\text { de texte s'agit-il? quel lien ont- } \\
\text { ils en commun et qu'est-ce qui } \\
\text { les différencie? } \\
\text { - Question: quelles sont les com- } \\
\text { pétences à mobiliser pour réussir } \\
\text { un bon compte-rendu? (lecture } \\
\text { ou audition/ expression orale ou } \\
\text { écrite) }\end{array}$ & 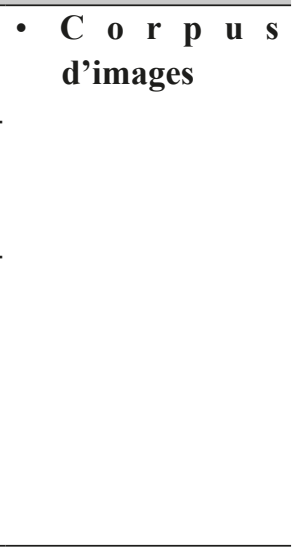 \\
\hline 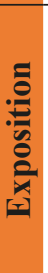 & $\begin{array}{l}\text { Faire découvrir une tech- } \\
\text { nique de lecture globale } \\
\text { (celle des } 5 \mathrm{~W} \text { ). Plutôt } \\
\text { que de choisir un texte } \\
\text { 'quelconque', ici il s'agit } \\
\text { de faire faire l'exercice }\end{array}$ & $\begin{array}{l}\text { - Surbased'untexteàlire,consigne: } \\
\text { remplissez le tableau du schéma } \\
\text { Quintilien (ou } 5 \text { W) }\end{array}$ & $\begin{array}{l}\text { - Texte X: com- } \\
\text { ment lire un } \\
\text { texte? } \\
\text { - Fichier avec le } \\
\text { tableau vide }\end{array}$ \\
\hline
\end{tabular}




\begin{tabular}{|c|c|c|c|}
\hline & Justification pédagogique & Déroulement & Ressources \\
\hline & $\begin{array}{l}\text { - Reprendre le texte tra- } \\
\text { vaillé en Exposition pour } \\
\text { vérifier la bonne compré- } \\
\text { hension du contenu } \\
\text { - Faire travailler les étu- } \\
\text { diants sur le lexique: } \\
\text { mots clefs, les idées ... } \\
\text { - Faire ressortir les élé- } \\
\text { ments à prendre en consi- } \\
\text { dération avant d'aborder } \\
\text { le texte, et les tâches fa- } \\
\text { cilitées par la maîtrise des } \\
\text { méthodes de lecture pour } \\
\text { pouvoir faciliter, par la } \\
\text { suite, les prises de notes }\end{array}$ & $\begin{array}{l}\text { - Favoriser l'échange sur le texte } \\
\text { - Vérifier le degré de compréhen- } \\
\text { sion du texte par la présentation, } \\
\text { sous forme schématique, d'infor- } \\
\text { mation conforme ou non au texte } \\
\text { - Faire dégager l'idée principale } \\
\text { du texte } \\
\text { - Repérer les mots clefs et ratta- } \\
\text { cher leur fonctionnement avec le } \\
\text { contenu global du texte } \\
\text { - Relever et classer dans un ta- } \\
\text { bleau les éléments d'identifica- } \\
\text { tion des étapes de lecture et les } \\
\text { relier et aux techniques et mé- } \\
\text { thodes de lecture utilisées. }\end{array}$ & $\begin{array}{l}\text { Ré i n vest is s e- } \\
\text { ment du texte: } \\
\text { - Texte X: com- } \\
\text { ment lire un } \\
\text { texte? } \\
\text { - Fiche outil sur } \\
\text { la prise de note } \\
\text { à mettre à la } \\
\text { disposition des } \\
\text { étudiants } \\
\text { - Fiche outil: } \\
\text { le schéma du } \\
\text { Quintilien (ou } \\
\text { le schéma des } 5 \\
\text { W) }\end{array}$ \\
\hline & $\begin{array}{l}\text { Effectuer une prise de } \\
\text { notes informelles à partir } \\
\text { de textes écrits }\end{array}$ & $\begin{array}{l}\text { - Faire lire les documents sur les- } \\
\text { quels les étudiants vont appli- } \\
\text { quer le schéma des }(5 \mathrm{~W}) \\
\text { - Faire comprendre que le recours } \\
\text { au schéma des (5W) est un outil } \\
\text { qui n'a d'efficacité que s'il est } \\
\text { utilisé minutieusement. Il aide à } \\
\text { faire gagner du temps et il n'est } \\
\text { pas une fin en soi. }\end{array}$ & $\begin{array}{l}\text { Textes à mettre } \\
\text { à la disposition } \\
\text { des étudiants }\end{array}$ \\
\hline
\end{tabular}

\section{Séquence 1}

Leçon 2: Traiter l'information: prendre des notes efficacement

\section{Compétences visées:}

$\sqrt{ }$ S'exercer aux techniques de lecture efficace

$\sqrt{ }$ Développer les réflexes d'analyse de documents de spécialité

$\sqrt{ }$ Se documenter et prendre des notes pour résumer, synthétiser, rendre compte $\cdots$

$\sqrt{ } \quad$ Utiliser le lexique approprié. 


\begin{tabular}{|c|c|c|c|}
\hline & Justification pédagogique & Déroulement & Ressources \\
\hline 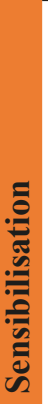 & $\begin{array}{l}\text { - Sensibiliser les étudiants } \\
\text { sur le rôle de la prise de } \\
\text { notes } \\
\text { Lire un document ou } \\
\text { écouter attentivement } \\
\text { une communication aca- } \\
\text { démique relève de princi- } \\
\text { paux réflexes et de com- } \\
\text { pétences à développer }\end{array}$ & $\begin{array}{l}\text { - Echange sur le rôle de la prise de } \\
\text { notes } \\
\text { Question: quelles sont les com- } \\
\text { pétences à mobiliser pour réussir } \\
\text { une prise de notes efficace? (lec- } \\
\text { ture efficace ou écoute attentive } \\
\text { - traitement/sélection d'informa- } \\
\text { tion) }\end{array}$ & $\begin{array}{l}\text { - Corpus de } \\
\text { textes à choisir }\end{array}$ \\
\hline 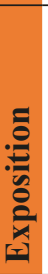 & $\begin{array}{l}\text { - Appréhender globale- } \\
\text { ment un texte } \\
\text { Exposer les différentes } \\
\text { techniques de prises de } \\
\text { notes }\end{array}$ & $\begin{array}{l}\text { Prendre des notes efficaces } \\
\text { Effectuer un balayage global du } \\
\text { texte: } \\
\sqrt{ } \text { Repérer les mots-clefs } \\
\sqrt{ } \text { Utiliser des symboles et des } \\
\text { abréviations }\end{array}$ & 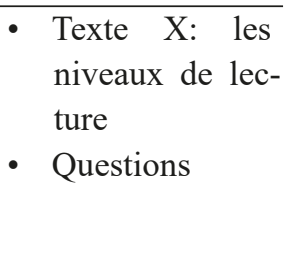 \\
\hline : & $\begin{array}{l}\text { - s'exercer à la prise de } \\
\text { notes }\end{array}$ & $\begin{array}{l}\text { - Synthèse sur les mots de liaison } \\
\text { - Application sur d'autres textes: } \\
\text { vérification la compréhension } \\
\text { des différentes techniques de } \\
\text { prise de notes }\end{array}$ & $\begin{array}{l}\text { - Réinvestisse- } \\
\text { ment }\end{array}$ \\
\hline 言 & $\begin{array}{l}\text { Utiliser des symboles } \\
\text { et des abréviations cou- } \\
\text { rantes dans le cadre d'une } \\
\text { prise de notes efficace }\end{array}$ & $\begin{array}{l}\text { - Faire lire les documents sur les- } \\
\text { quels les étudiants vont effectuer } \\
\text { des prises de notes } \\
\text { - Faire comprendre que le recours } \\
\text { à la prise de notes est un outil qui } \\
\text { aide à faire gagner du temps et il } \\
\text { n'est pas une fin en soi. } \\
\text { - La répétition de l'exercice de } \\
\text { prise de notes permet de maîtri- } \\
\text { ser les mécanismes et réflexes } \\
\text { judicieux dans le cadre de la } \\
\text { lecture compréhension de docu- } \\
\text { ments. }\end{array}$ & $\begin{array}{l}\text { - Auto-évalua- } \\
\text { tion et échanges } \\
\text { de productions } \\
\text { et corrections } \\
\text { par les pairs }\end{array}$ \\
\hline
\end{tabular}


